\title{
Flattening and surface-brightness of the fast-rotating star $\delta$ Persei with the visible VEGA/CHARA interferometer
}

\author{
M. Challouf ${ }^{2,1}$, N. Nardetto ${ }^{1}$, A. Domiciano de Souza $^{1}$, D. Mourard ${ }^{1}$, I. Tallon-Bosc ${ }^{3}$, H. Aroui ${ }^{2}$, C. Farrington ${ }^{4}$, \\ R. Ligi ${ }^{5}$, A. Meilland ${ }^{1}$, and M. Mouelhi ${ }^{2}$ \\ 1 Université Côte d'Azur, Observatoire de la Côte d'Azur, CNRS, Laboratoire Lagrange, UMR 7293, 06304 Nice Cedex 4, France \\ e-mail: mounir.challouf@oca.eu \\ ${ }^{2}$ Laboratoire Dynamique Moléculaire et Matériaux Photoniques, UR11ES03, Université de Tunis/ENSIT, Tunis, Tunisie \\ 3 Université Lyon1, ENS Lyon, CNRS, Centre de Recherche Astrophysique de Lyon UMR 5574, 69230 Saint-Genis-Laval, France \\ 4 CHARA Array, Mount Wilson Observatory, 91023, Mount Wilson, Pasadena, CA 91109, USA \\ 5 Aix Marseille Université, CNRS, LAM (Laboratoire d'Astrophysique de Marseille), UMR 7326, 13388 Marseille, France
}

Received 20 October 2016 / Accepted 26 April 2017

\begin{abstract}
Context. Rapid rotation is a common feature for massive stars, with important consequences on their physical structure, flux distribution and evolution. Fast-rotating stars are flattened and show gravity darkening (non-uniform surface intensity distribution). Another important and less studied impact of fast-rotation in early-type stars is its influence on the surface brightness colour relation (hereafter SBCR), which could be used to derive the distance of eclipsing binaries.

Aims. The purpose of this paper is to determine the flattening of the fast-rotating B-type star $\delta$ Per using visible long-baseline interferometry. A second goal is to evaluate the impact of rotation and gravity darkening on the $V-K$ colour and surface brightness of the star.

Methods. The B-type star $\delta$ Per was observed with the VEGA/CHARA interferometer, which can measure spatial resolutions down to 0.3 mas and spectral resolving power of 5000 in the visible. We first used a toy model to derive the position angle of the rotation axis of the star in the plane of the sky. Then we used a code of stellar rotation, CHARRON, in order to derive the physical parameters of the star. Finally, by considering two cases, a static reference star and our best model of $\delta$ Per, we can quantify the impact of fast rotation on the surface brightness colour relation (SBCR).

Results. We find a position angle of $23 \pm 6$ degrees. The polar axis angular diameter of $\delta$ Per is $\theta_{\mathrm{p}}=0.544 \pm 0.007$ mas, and the derived flatness is $r=1.121 \pm 0.013$. We derive an inclination angle for the star of $i=85_{-20}^{+5}$ degrees and a projected rotation velocity $V \sin i=175_{-11}^{+8} \mathrm{~km} \mathrm{~s}^{-1}$ (or $57 \%$ of the critical velocity). We find also that the rotation and inclination angle of $\delta$ Per keeps the $V-K$ colour unchanged while it decreasing its surface-brightness by about 0.05 mag.

Conclusions. Correcting the impact of rotation on the SBCR of early-type stars appears feasible using visible interferometry and dedicated models.
\end{abstract}

Key words. stars: early-type - stars: rotation - methods: data analysis - instrumentation: interferometers techniques: interferometric

\section{Introduction}

The equilibrium structure of a rotating star is achieved by the interplay of three forces: the usual gravity and force of pressure, and an additional one, the centrifugal force. Because this centrifugal force depends on latitude (zero at the poles and maximum at the equator) the stellar photosphere is not spherical but becomes flattened, resembling a two-axis ellipsoid (see for example Kopal 1987). Rapid rotation also induces a gravity darkening, that is a flux (effective temperature) gradient from the poles to the equator of the star (von Zeipel 1924). Both deformation and gravity darkening affect the distribution of the surface brightness at the star photosphere (Challouf et al. 2015a,b). Another consequence linked to the existence of gravity darkening and rotation is the creation inside the star of a slow flow of matter (e.g. Rieutord 2006). This flow has a multitude effect on the star properties, such as: i) suppling the core with hydrogen and thus increase the lifetime of the star; ii) enriching the outer layers with metals; iii) transporting the angular moment of the core to the surface and thus increase the rotational velocity of the photosphere (Meynet \& Maeder 2000; Ekström et al. 2008).

Fast rotating stars and their environments are known to have an impact on the calibration of SBCR of early-type stars, limiting the precision on the relation to around 0.16 mag (Challouf et al. 2014b). The SBCR is necessary to derive the distance of early-type eclipsing binaries $(\mathrm{O}, \mathrm{B}, \mathrm{A})$, which are much brighter and thus easier to detect than late-type eclipsing binaries (Pietrzyński et al. 2009; Mochejska et al. 2001; Vilardell et al. 2006; Pawlak et al. 2013). However, to achieve 1\% precision in term of distances or 0.02 mag of precision on the SBCR, one has to consider massive star properties, such as rotation. In this context $\delta$ Per is a good target to study the effects of the rapid rotation.

In Sect. 2, we present our VEGA/CHARA observations and data processing and show that the fast rotating star $\delta$ Per is currently in its B-type phase. In Sect. 3, we analyze our data with 
Table 1. Journal of observations.

\begin{tabular}{|c|c|c|c|}
\hline Date & UT & Configuration & Cal. \\
\hline 2014-08-30 & $\begin{array}{l}09: 07,09: 39,10: 14 \\
10: 48,11: 17,11: 50\end{array}$ & W2S2W1 & 1 \\
\hline $2013-11-28$ & $\begin{array}{l}02: 51,03: 10,03: 25 \\
03: 40,04: 41,04: 55 \\
05: 11,05: 27,05: 41\end{array}$ & W2S2 & $2,3,4$ \\
\hline 2013-10-26 & $07: 47$ & E2E1W2 & 1 \\
\hline 2013-10-25 & $\begin{array}{c}06: 59,07: 10,11: 20 \\
11: 49,12: 17\end{array}$ & E2E1W2 & 1,5 \\
\hline 2013-10-24 & 07:27, 07:52 & E2S1W2 & 1,5 \\
\hline 2012-08-30 & $09: 48,10: 30,11: 07$ & W2W1E2 & 1 \\
\hline
\end{tabular}

Table 2. Reference stars and their parameters, including the $m_{V}$ and $m_{k}$ magnitudes, and the predicted uniform disc angular diameter (in mas) in the $R$ band.

\begin{tabular}{lcccc}
\hline \hline $\begin{array}{l}\text { No. } \\
\text { Cal. }\end{array}$ & $\begin{array}{c}\text { Reference } \\
\text { stars }\end{array}$ & $\begin{array}{c}m_{V} \\
{[\mathrm{mag}]}\end{array}$ & $\begin{array}{c}m_{K} \\
{[\mathrm{mag}]}\end{array}$ & $\begin{array}{c}\theta_{\mathrm{UD}}[R] \\
{[\mathrm{mas}]}\end{array}$ \\
\hline 1 & HD 18411 & 4.677 & 4.418 & $0.400 \pm 0.029$ \\
2 & HD 25642 & 4.272 & 4.149 & $0.439 \pm 0.031$ \\
3 & HD 25940 & 4.000 & 3.796 & $0.536 \pm 0.038$ \\
4 & HD 20677 & 4.940 & 4.776 & $0.329 \pm 0.024$ \\
5 & HD 27396 & 4.813 & 4.886 & $0.281 \pm 0.020$ \\
\hline
\end{tabular}

different models and in Sect. 4, we compare the $V-K$ colour and surface brightness of a static reference star (without flattening) with our best model of $\delta$ Per.

\section{VEGA/CHARA observations of $\delta$ Per}

The Center for High Angular Resolution Astronomy (CHARA) array operates in the visible and the near-infrared (ten Brummelaar et al. 2005). Located at the Mount Wilson Observatory, north of Los Angeles (California, USA), the CHARA Array consists of six 1-m telescopes in a non-redundant Yshaped configuration that offers 15 different baselines between 34 and $331 \mathrm{~m}$. The Visible spEctroGraph and polArimeter (VEGA; Mourard et al. 2009) is one of the beam combiners installed on the CHARA Array. It works in the visible and can combine up to four different telescopes (Mourard et al. 2011). With the longest baseline, in the visible domain, one can reach a spatial resolution of 0.3 mas (and 1.6 mas in the near-infrared).

$\delta$ Per was observed with VEGA/CHARA over a three year period from August 2012 to December 2014 (Table 1). An interferometric observation requires the acquisition of a number of calibrators before and after the target (Challouf et al. 2012, 2014a). To provide a better estimation of the transfer function during the observations of the target, each measurement was calibrated using stars listed in Table 2 with a sequence C-T-C (C referring to the calibrator and $\mathrm{T}$ to the science target), several times in a row when possible. These calibrators were selected using the SearchCal ${ }^{1}$ software developed by the Jean-Marie Mariotti Center (JMMC; Bonneau et al. 2006). Six different telescopes configurations taken at different hour angles allowed us to obtain measurements well distributed over the $(u, v)$ plane (see Table 1 and Fig. 1). We also used two spectral bands of $\Delta \lambda=20 \mathrm{~nm}$ centered on $\lambda=715 \mathrm{~nm}$ and $\lambda=735 \mathrm{~nm}$, in the medium spectral resolving power mode of VEGA $(R=5000)$. In Fig 2 , we

\footnotetext{
1 Available at http: //WwW . jmmc. fr/searchcal
}

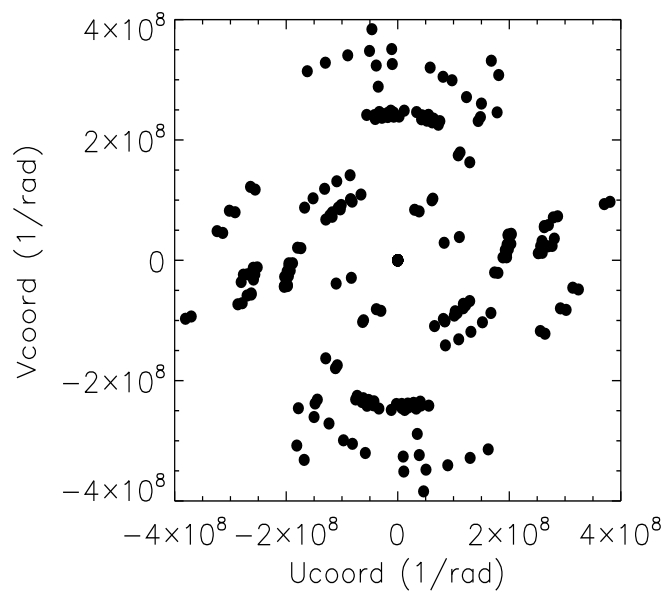

Fig. 1. $(u-v)$ plane coverage of VEGA observations.

show also one of our $\mathrm{H} \alpha$ line profile of $\delta$ Per. This profile is in absorption, without any signature of a circumstellar disc, which means that $\delta$ Per was not active at the time of observations (i.e. between 2012 and 2014), which is consistent with the ELODIE ${ }^{2}$ spectra obtained in 1997. Thus our observations show that $\delta$ Per is a B-type star, which allows an accurate determination of its rotation (i.e. without contamination by a circumstellar disc).

Our VEGA measurements corresponds to 30 blocks of observations, each block containing 2500 images of ten milliseconds. For each block, the raw squared visibility is estimated using the auto-correlation mode (Mourard et al. 2009, 2011). The sequence of calibrators permits the estimate of the transfer function and then allows derivation the calibrated visibility of the target $\left(V_{\text {target }}\right)$. The visibility systematic uncertainties that stem from the uncertainty on the reference stars are calculated according to the method described in Mourard et al. (2012) and presented in details in Appendix A. In our case, in the worse situation, we found a systematic error of 0.008 compared to a statistical error of 0.062 . Therefore we consider these systematic errors as negligible in our study. Fabricius \& Makarov (2000) shows that $\delta$ Per is a binary star. It has an optical companion with an apparent magnitude of +6.17 at an angular separation of 0.330 arcsec and a position angle of $221^{\circ}$. Given the large difference of magnitude and the separation between the two components, the binarity of $\delta$ Per does not impact our data.

\section{Modelling of $\delta$ Per}

\subsection{A toy model}

The calibrated visibilities obtained for each observation are used to constrain a model of uniform elongated disc, with only three geometrical parameters: the minor axis diameter or polar diameter $\theta_{\mathrm{p}}^{\prime}$ (apparent diameter), the elongation ratio $r^{\prime}$ defined along the major axis as the ratio between the major apparent diameter and the minor one, and the position angle $\phi$ of the rotation axis of the star (i.e. the minor axis), equal to the angle between the positive vertical semi-axis (i.e. north direction) and the major axis towards to the positive horizontal semi-axis (i.e. east direction). This is performed using the LITpro ${ }^{3}$ software developed by the JMMC (Tallon-Bosc et al. 2008). We find $\theta_{\mathrm{p}}^{\prime}=$ $0.548 \pm 0.008$ mas, $\phi=23 \pm 6$ degrees and $r^{\prime}=1.115 \pm 0.024$

\footnotetext{
2 Available at http://atlas.obs-hp.fr/elodie/

3 Available at http://www. jmmc. fr/litpro
} 


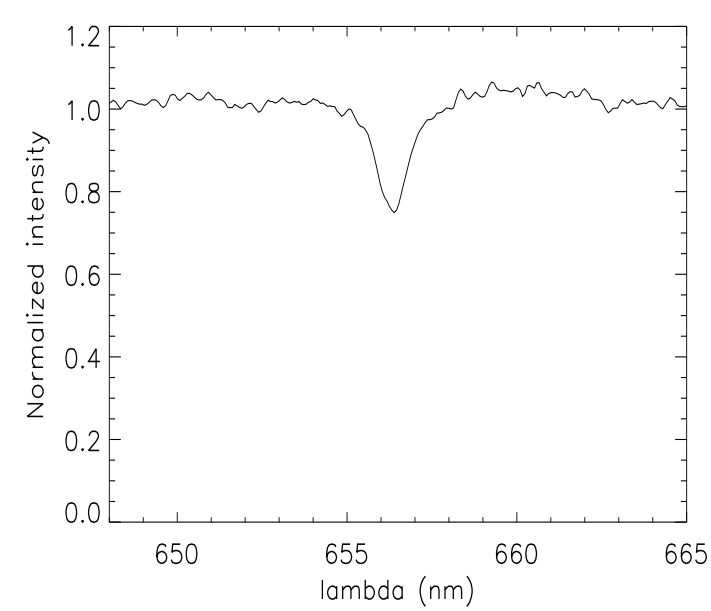

Fig. 2. VEGA/CHARA normalized spectrum of $\delta$ Per $(R \simeq 5000)$ around the $\mathrm{H}_{\alpha}$ spectral line.

(with a reduced $\chi^{2}$ of 2.7 , while we obtain 3.8 if we fit a uniform disc to the data). The elongation of $\delta$ Per is thus detected $($ at $5 \sigma)$ and refined in next section using a dedicated model. $\theta_{\mathrm{p}}^{\prime}$ is consistently lower than the mean spectrophotometric diameter of $0.583 \pm 0.013$ mas found by Zorec et al. (2009), while the equatorial diameter $(0.611 \pm 0.022 \mathrm{mas})$ is indeed larger. The position angle $\phi$, which is uncorrelated with the two other parameters, is used in the next subsection in order to constrain the dedicated model of $\delta$ Per.

\subsection{A fast rotating star model: CHARRON}

The second step of the modelling was done with the code for high angular resolution of rotating objects in nature (CHARRON) described by Domiciano de Souza et al. (2012a,b, 2002). The stellar photospheric shape is given by the commonly adopted Roche approximation, which is well adapted to nondegenerate, fast-rotating stars. The effective temperature $\left(T_{\text {eff }}\right)$ at the surface of fast rotators is not uniform (depending on the colatitude $\theta$ ) owing to the decreasing effective gravity $\left(g_{\text {eff }}-\right.$ gravitation plus centrifugal acceleration) from the poles to the equator (gravity darkening effect). We modelled the gravity darkening as a generalized form of the von Zeipel law (von Zeipel 1924):

$T_{\text {eff }}(\theta)=K g_{\text {eff }}^{\beta}(\theta)$,

where $\beta$ is the gravity darkening coefficient. von Zeipel (1924) derived a theoretical value of 0.25 for early-type stars with radiative external layers and pressure, only depending on the density (barotropic approximation). However, recent interferometric observations have measured somewhat lower values for $\beta$ (e.g. Che et al. 2011; Domiciano de Souza et al. 2014). The value of 0.20 seems a good compromise between the theoretical value of von Zeipel and the values measured from interferometric observations of fast-rotating stars and also with theoretical models of gravity darkening (e.g. Espinosa Lara \& Rieutord 2011; Claret 2012). We thus considered $\beta=0.20$ for our model of $\delta$ Per.

The $K$ quantity is the proportionality constant between $T_{\text {eff }}(\theta)$ and $g_{\text {eff }}(\theta)$. It depends on the stellar physical input parameters of the model, which are: the mean effective temperature $\left(T_{\text {eff }}\right)$, the mass $(\mathrm{M})$, and the equatorial radius $\left(R_{\text {eq }}\right)$. Table 3 lists the fundamental parameters considered as an input to the model, together with their corresponding references. In addition to these parameters, the orientation in the plane of the sky $(\phi)$ was fixed to 23 degrees as found in Sect. 3.1 using the toy model.

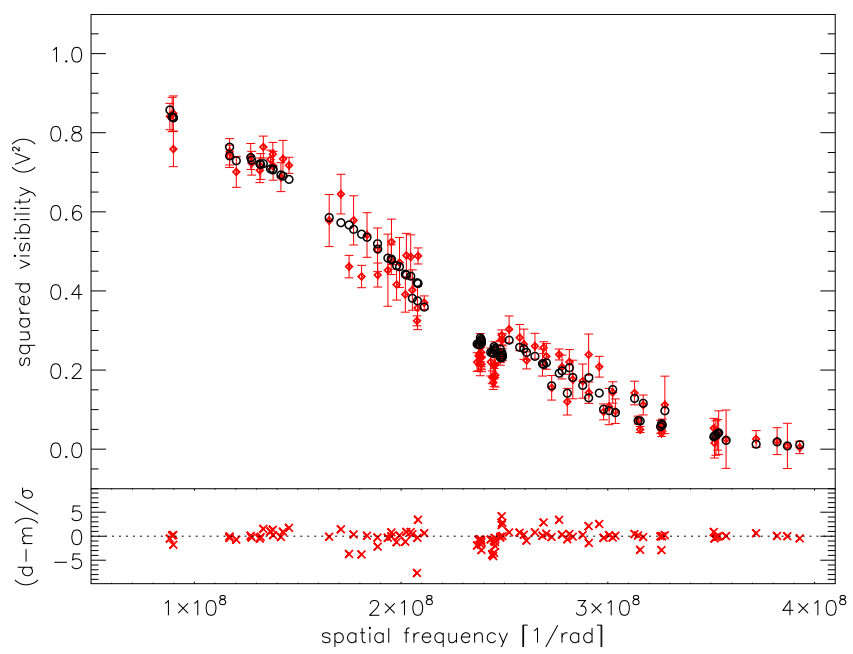

Fig. 3. Calibrated VEGA/CHARA squared visibilities are plotted as a function of the spatial frequency (red points). The open black circles are the synthetic visibilities obtained from the FFT of the image of $\delta$ Per obtained with the CHARRON code. The bottom panel shows the residuals of the visibilities.

We basically fitted two parameters: the inclination $(i)$ and the rotation velocity $V_{\text {rot }}$. This was done by considering different values of $i \in\left[0^{\circ}, 90^{\circ}\right]$ and $V_{\text {rot }} \in\left[0 V_{\mathrm{c}}, 0.95 V_{\mathrm{c}}\right]$ with steps of $5^{\circ}$ and $3 \mathrm{~km} \mathrm{~s}^{-1}$, respectively. For each model, we computed an intensity distribution of the star using the ATLAS9 stellar atmosphere models (Kurucz 1979) and the spectral synthesis code SYNSPEC (Hubeny \& Lanz 2011). To compare the theoretical and observed calibrated squared visibilities, we calculate the fast Fourier transform (FFT hereafter) of this intensity distribution following the theorem of Zernike-Van Cittert (van Cittert 1934; Zernike 1938). We calculate for each model a reduced $\chi^{2}$ and find that the best parameters (with a reduced $\chi^{2}$ of 2.8) are: $i=85_{-20}^{+5}$ degrees and $V_{\text {rot }}=176_{-6}^{+7} \mathrm{~km} \mathrm{~s}^{-1}$ (or $57 \%$ of the critical velocity). The derived projected rotational velocity is consistent with previous studied: $V_{\text {rot }} \sin i=200 \pm 40 \mathrm{~km} \mathrm{~s}^{-1}$ (Slettebak et al. 1975; Wolff et al. 1982) and $190 \pm 24 \mathrm{~km} \mathrm{~s}^{-1}$ (Abt et al. 2002). The CHARRON model provides the true parameters (i.e. not apparent), taking into account the gravity darkening. We find: $\theta_{\mathrm{p}}=0.544 \pm 0.007$ mas and $r=1.121 \pm 0.013$. The synthetic intensity distribution of the star with the observed and computed calibrated squared visibilities are plotted in Fig. 3. The final parameter values for the best-fit model are presented in Table 3. From the $\theta_{\mathrm{p}}$ and $\theta_{\mathrm{e}}$ (in mas) and the parallax $\pi$ (in second of arc), we estimated the linear radius and its error by using a simple Monte Carlo simulation:

$R_{(\mathrm{p}, \mathrm{e})} \pm \delta R\left(R_{\odot}\right)=\frac{\theta_{(\mathrm{p}, \mathrm{e})} \pm \delta \theta_{(\mathrm{p}, \mathrm{e})}}{9.305 \times(\pi \pm \delta \pi)}$,

we obtain finally a polar radius of $R_{\mathrm{p}}=9.25 \pm 1.60 R_{\odot}$ and an equatorial radius of $R_{\mathrm{e}}=10.39 \pm 1.60 R_{\odot}$. We note that the uncertainty on the radius is dominated by the parallax uncertainty.

\section{Impact of the rotation on the $V-K$ colour and surface brightness of $\delta$ Per}

We derived the $V-K$ and $\mathrm{Sv}$ following the methodology described below and detailed in Challouf et al. (2014c), Challouf et al. (2015b). The surface brightness is defined as $S_{\lambda}=m_{\lambda}+5 \log \theta_{\mathrm{LD}}$, with $m_{\lambda}$ the absolute visual magnitude and $\theta_{\mathrm{LD}}$ the limb-darkened angular diameter of the star. 


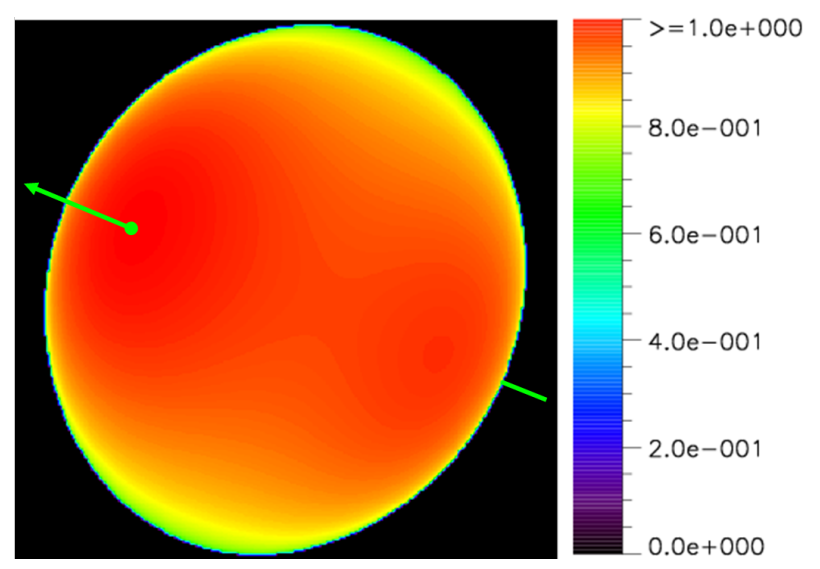

Fig. 4. Intensity map of the best CHARRON model of $\delta$ Per. The flatness of the star is of $r=1.121$ with an inclination angle of $i=85^{\circ}$ and an rotation velocity of $57 \%$ of $V_{c}$. The image is given in units of the equatorial radius, $0.611 \mathrm{X} 0.611$ mas.

Table 3. Physical parameters of $\delta$ Per.

\begin{tabular}{lrc}
\hline \hline $\begin{array}{l}\text { Model } \\
\text { parameters }\end{array}$ & \multicolumn{1}{c}{ Values } & Unity \\
\hline$T_{\text {mean }}{ }^{a}$ & $14890^{\star}$ & $K$ \\
$M^{b}$ & $7.0^{\star}$ & $M_{\odot}$ \\
$R_{\text {eq }}{ }^{c}$ & $10.5^{\star}$ & $R_{\odot}$ \\
$\beta$ & $0.20^{\star}$ & \\
$\phi$ & $23^{\star}$ & $\mathrm{deg}$ \\
\hline$i$ & $i=85_{-20}^{+5 \star}$ & $\mathrm{deg}$ \\
$V_{\text {rot }}$ & $176_{-6}^{+7 \star}$ & $\mathrm{km} \mathrm{s}^{-1}$ \\
$\theta_{\mathrm{p}}$ & $0.544 \pm 0.007^{\star \star}$ & $\mathrm{mas}$ \\
$r$ & $1.121 \pm 0.013^{\star \star}$ & \\
\hline$\chi^{2}$ & 2.8 & \\
\hline
\end{tabular}

Notes. The asterisk indicates the input CHARRON models parameters (Domiciano de Souza et al. 2012a,b, 2002). The double asterisk indicates the parameters obtained with the best CHARRON model.

References. ${ }^{(a)}$ Zorec et al. (2009); ${ }^{(b)}$ Code et al. (1976); ${ }^{(c)}$ Underhill et al. (1979).

The photometric magnitudes in the $V$ and $K$ bands are derived from the CHARRON model, following the method described in Domiciano de Souza et al. (2014). The squared visibilities $\left(V^{2}\right)$ obtained from the intensity distribution (previous section) are then interpreted using a simple uniform disc model. The equivalent uniform disc angular diameter $\theta_{\mathrm{UD}}$ was then converted into a limb-darkened disc using the relation of Hanbury Brown et al. (1974).

The value of the surface-brightness is, by construction, biased by the rotation of the star. The $V-K$ colour is an output of the model, and is also impacted by the rotation. This procedure is done for our best model of $\delta$ Per indicated in Fig. 5. Also indicated in Fig. 5 is the corresponding static model (i.e. without rotation) considering the physical input parameters (Table 3 ). We can make the following specific remarks:

1. The static and the best model of $\delta$ Per have the same $V-K$ colour.

2. The rotation and inclination of $\delta$ Per decrease its surfacebrightness (compared to a static model) by about 0.05 mag.

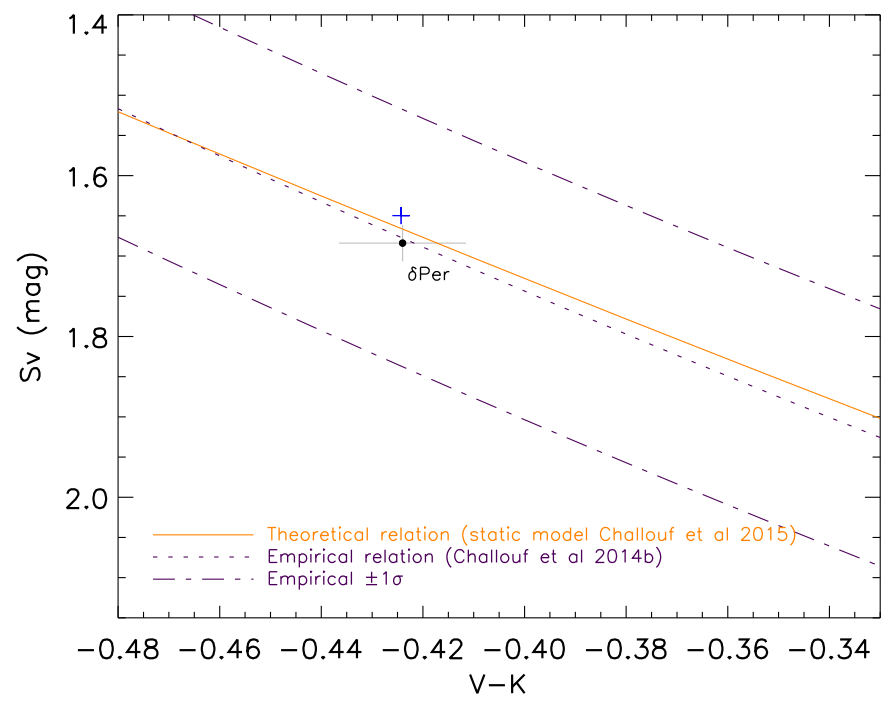

Fig. 5. The surface brightness for the CHARRON model is plotted versus the $V-K$ colour in order to illustrate the impact of the rotation and inclination (see the text).

3. This bias of 0.05 mag found for $\delta$ Per is much lower than the $1 \sigma$ intrinsic dispersion (or $0.16 \mathrm{mag}$ ) found on the SBCR by Challouf et al. (2014b), and is consistent with the bias on the SBCR (offset and dispersion) due to rotation estimated in Challouf et al. (2015b).

\section{Conclusions}

We derive here, for the first time, the flatness of $\delta$ Per $(r=$ $1.121 \pm 0.013)$ using the visible VEGA/CHARA interferometer and the CHARRON model. The derived rotation velocity is $V_{\text {rot }}=176_{-6}^{+7} \mathrm{~km} \mathrm{~s}^{-1}$ (or $57 \%$ of the critical velocity). We find that the rotation of $\delta$ Per (combined with its inclination angle of $i=85_{-20}^{+5}$ degrees) has no impact on the derived $V-K$ colour while it reduces its surface brightness of 0.05 mag. We conclude that $\delta$ Per, as many fast rotators should not be used in order to calibrate the SBCR at the 0.05 mag level (or 2.5\%) for early-type stars (Challouf et al. 2015b), unless a correction in the $V-K$ colour and the surface brightness is done based on a dedicated modelling.

Acknowledgements. The authors acknowledge financial support from "Programme National de Physique Stellaire" (PNPS) of CNRS/INSU, France. This work is based upon observations obtained with the Georgia State University Center for High Angular Resolution Astronomy Array at Mount Wilson Observatory. The CHARA Array is supported by the National Science Foundation under Grant No. AST-1211929. Institutional support has been provided from the GSU College of Arts and Sciences and the GSU Office of the Vice President for Research and Economic Development. This research has made use of the Jean-Marie Mariotti Center Aspro service ${ }^{1}$, and the electronic bibliography maintained by the NASA/ADS system. This research has made use of the Jean-Marie Mariotti Center SearchCal service ${ }^{2}$ co-developed by FIZEAU and LAOG/IPAG, and CDS Astronomical Databases SIMBAD and VIZIER ${ }^{3}$. This research has made use of the Jean-Marie Mariotti Center LITpro service codeveloped by CRAL, IPAG, and LAGRANGE ${ }^{4}$. This research has made use of the Jean-Marie Mariotti Center OiDB service 5 .

Available at http://www . jmmc. fr/aspro

Available at http://www . jmmc. fr/searchcal

Available at http://cdsweb.u-strasbg.fr/

4 LITpro software available at http://www . jmmc . fr/litpro

5 Available at http://oidb.jmmc.fr 
M. Challouf et al.: Study of $\delta$ Per of the fast-rotating star with VEGA/CHARA interferometer

\section{References}

Abt, H. A., Levato, H., \& Grosso, M. 2002, ApJ, 573, 359

Bonneau, D., Clausse, J.-M., Delfosse, X., et al. 2006, A\&A, 456, 789

Challouf, M., Nardetto, N., Mourard, D., Aroui, H., \& Chesneau, O. 2012 in SF2A-2012: Proc. Annual meeting French Society of Astronomy and Astrophysics, eds. S. Boissier, P. de Laverny, N. Nardetto, et al., 299

Challouf, M., Nardetto, N., Mourard, D., Aroui, H., \& Delaa, O. 2014a, in SF2A2014: Proc. Annual meeting French Soc. Astron. Astrophys., eds. J. Ballet, F. Martins, F. Bournaud, R. Monier, \& C. Reylé, 471

Challouf, M., Nardetto, N., Mourard, D., et al. 2014b, A\&A, 570, A104

Challouf, M., Nardetto, N., De Souza, A., et al. 2014c, Proc. Int. Astron. Union, 9, 288

Challouf, M., Nardetto, N., Domiciano de Souza, A., et al. 2015a, in IAU Symp., 307,288

Challouf, M., Nardetto, N., Domiciano de Souza, A., et al. 2015b, A\&A, 579, A107

Che, X., Monnier, J. D., Zhao, M., et al. 2011, ApJ, 732, 68

Claret, A. 2012, A\&A, 538, A3

Code, A. D., Bless, R. C., Davis, J., \& Brown, R. H. 1976, ApJ, 203, 417

Domiciano de Souza, A., Vakili, F., Jankov, S., Janot-Pacheco, E., \& Abe, L. 2002, A\&A, 393, 345

Domiciano de Souza, A., Hadjara, M., Vakili, F., et al. 2012a, A\&A, 545, A130

Domiciano de Souza, A., Zorec, J., \& Vakili, F. 2012b, in SF2A-2012: Proc. Annual meeting French Soc. Astron. Astrophys., eds. S. Boissier, P. de Laverny, N. Nardetto, R. Samadi, D. Valls-Gabaud, \& H. Wozniak, 321

Domiciano de Souza, A., Kervella, P., Moser Faes, D., et al. 2014, A\&A, 569, A10

Ekström, S., Meynet, G., Chiappini, C., Hirschi, R., \& Maeder, A. 2008, A\&A, 489,685

Espinosa Lara, F., \& Rieutord, M. 2011, A\&A, 533, A43

Fabricius, C., \& Makarov, V. V. 2000, A\&A, 356, 141
Hanbury Brown, R., Davis, J., Lake, R. J. W., \& Thompson, R. J. 1974, MNRAS, 167,475

Hubeny, I., \& Lanz, T. 2011, Astrophysics Source Code Library [record ascl: 1109.022]

Kopal, Z. 1987, Ap\&SS, 133, 157

Kurucz, R. L. 1979, ApJS, 40, 1

Meynet, G., \& Maeder, A. 2000, A\&A, 361, 101

Mochejska, B. J., Kaluzny, J., Stanek, K. Z., \& Sasselov, D. D. 2001, AJ, 122, 1383

Mourard, D., Clausse, J. M., Marcotto, A., et al. 2009, A\&A, 508, 1073

Mourard, D., Bério, P., Perraut, K., et al. 2011, A\&A, 531, A110

Mourard, D., Challouff, M., Ligi, R., et al. 2012, in Performance, results, and prospects of the visible spectograph VEGA on CHARA, SPIE Conf. Ser., 8445, 84450K

Pawlak, M., Graczyk, D., Soszyński, I., et al. 2013, Acta Astron., 63, 323

Pietrzyński, G., Thompson, I. B., Graczyk, D., et al. 2009, ApJ, 697, 862

Rieutord, M. 2006, A\&A, 451, 1025

Slettebak, A., Collins, II, G. W., Parkinson, T. D., Boyce, P. B., \& White, N. M. 1975, ApJS, 29, 137

Tallon-Bosc, I., Tallon, M., Thiébaut, E., et al. 2008, in LITpro: a model Fitting software for optical interferometry, SPIE Conf., 7013, 7013J

ten Brummelaar, T. A., McAlister, H. A., Ridgway, S. T., et al. 2005, ApJ, 628, 453

Underhill, A. B., Divan, L., Prevot-Burnichon, M.-L., \& Doazan, V. 1979, MNRAS, 189, 601

van Cittert, P. H. 1934, Physica, 1, 201

Vilardell, F., Ribas, I., \& Jordi, C. 2006, A\&A, 459, 321

von Zeipel, H. 1924, MNRAS, 84, 665

Winzer, P. J. 2000, Rev. Sci. Instr., 71, 1447

Wolff, S. C., Edwards, S., \& Preston, G. W. 1982, ApJ, 252, 322

Zernike, F. 1938, Physica, 5, 785

Zorec, J., Cidale, L., Arias, M. L., et al. 2009, A\&A, 501, 297 


\section{Appendix A: Estimation of the error on the calibrated squared visibilities}

The squared visibility of the target may be written as follows:

$V_{\text {target }}^{2}=V_{\text {cal }}^{2} \frac{\mu_{\text {target }}^{2}}{\mu_{\text {cal }}^{2}}$,

where $V_{\text {target }}^{2}$ and $V_{\text {cal }}^{2}$ are the true squared visibility of the target and the calibrator respectively, and $\mu_{\text {target }}^{2}$ and $\mu_{\text {cal }}^{2}$ their measured squared visibility. This equation assumes the constancy of the transfer function between the observations of the target and the calibrator.

The three terms of the right side of the equality are independent random variables. Whereas the product of two random variables, as $V_{\text {cal }}^{2} \mu_{\text {target }}^{2}$, is calculable, the quotient of two random variables, here $V_{\text {cal }}^{2} \mu_{\text {target }}^{2}$ by $\mu_{\text {cal }}^{2}$, follows a law which does not have moments: neither the mean nor the variance is calculable and both must be estimated approximately. The approximation can be of a different order, depending on the value of the signalto-noise ratio $(\mathrm{S} / \mathrm{N})$ of the denominator, meaninf here on $\mu_{\mathrm{cal}}^{2}$. The $\mathrm{S} / \mathrm{N}$ of a random variable is equal to the ratio of its mean on its standard deviation (or square root of its variance). To simplify the writing, we call $\alpha, \beta, \gamma$ the $\mathrm{S} / \mathrm{N}$ of $\mu_{\text {target }}^{2}, \mu_{\text {cal }}^{2}$ and $V_{\text {cal }}^{2}$ :

$\alpha=\frac{\left\langle\mu_{\text {target }}^{2}\right\rangle}{\sigma_{\mu_{\text {target }}^{2}}}, \quad \beta=\frac{\left\langle\mu_{\text {cal }}^{2}\right\rangle}{\sigma_{\mu_{\text {cal }}^{2}}}, \quad \gamma=\frac{\left\langle V_{\text {cal }}^{2}\right\rangle}{\sigma_{V_{\text {cal }}^{2}}}$,

$<>$ denoting the mean and $\sigma$ the standard deviation. Using the study of Winzer (2000) and the independence of the variables (cancelling covariance-terms), we found, if $\beta$ is larger than four, the following second order approximation with about $1 \%$ accuracy of both first moments of the quotient of Eq. (A.1):

$$
\begin{aligned}
& \left\langle V_{\text {target }}^{2}\right\rangle \simeq\left\langle V_{\text {cal }}^{2}\right\rangle^{2} \frac{\left\langle\mu_{\text {target }}^{2}\right\rangle^{2}}{\left\langle\mu_{\text {cal }}^{2}\right\rangle^{2}}\left[1+\frac{1}{\beta^{2}}\right], \\
& \sigma_{V_{\text {target }}^{2}}^{2} \simeq\left\langle V_{\text {target }}^{2}\right\rangle^{2}\left[\frac{1}{\alpha^{2}}+\frac{1}{\beta^{2}}+\frac{1}{\gamma^{2}}+\frac{1}{\beta^{2} \gamma^{2}}-\frac{1}{\beta^{4}}\right],
\end{aligned}
$$

Eq. (A.3) shows that the quadratic error on $V_{\text {target }}^{2}$ may be written as the sum of two terms, one of those being independent of $\gamma$, which we call statistic quadratic error. The second term is named systematic quadratic error:

$$
\begin{aligned}
\sigma_{V_{\text {target stat }}^{2}}^{2} \simeq\left\langle V_{\text {cal }}^{2}\right\rangle^{2} \frac{\left\langle\mu_{\text {target }}^{2}\right\rangle^{2}}{\left\langle\mu_{\text {cal }}^{2}\right\rangle^{2}}\left[\frac{1}{\alpha^{2}}+\frac{1}{\beta^{2}}-\frac{1}{\beta^{4}}\right], \\
\sigma_{V_{\text {target syst }}^{2}}^{2} \simeq\left\langle V_{\text {cal }}^{2}\right\rangle^{2} \frac{\left\langle\mu_{\text {target }}^{2}\right\rangle^{2}}{\left\langle\mu_{\text {cal }}^{2}\right\rangle^{2}} \frac{1}{\gamma^{2}}\left[1+\frac{1}{\beta^{2}}\right] .
\end{aligned}
$$

\section{A.1. Influence of the true visibility of the calibrator}

$V_{\text {cal }}^{2}$ is a statistical variable whose mean is the squared visibility related to the supposed geometrical shape of the calibrator. If this is a uniform disc of diameter $\theta_{\mathrm{UD}}$, the relation is:

$\left\langle V_{\text {cal }}^{2}\right\rangle=4\left(\frac{J_{1}(z)}{z}\right)^{2}$

with $z=\pi\left\langle\theta_{\mathrm{UD}}\right\rangle B / \lambda$ where $B$ is the baseline and $\lambda$ the wavelength. $\left\langle\theta_{\mathrm{UD}}\right\rangle$ is given for example by SearchCal (Bonneau et al. 2006) after a polynomial fit in the (angular diameter, colour indices) space with a standard deviation at one $\sigma$. We have numerically shown that, if $\gamma$ is larger than four, $\gamma$ is proportional to the SNR of $\theta_{\mathrm{UD}}$ as follows:

$\frac{1}{\gamma}=2 z\left|\frac{J_{2}(z)}{J_{1}(z)}\right| \frac{\sigma \theta_{\mathrm{UD}}}{\left\langle\theta_{\mathrm{UD}}\right\rangle}$.

Equations (A.6) and (A.7) are then inserted into the final equations. We may verify that if the calibrator is well known, i.e. with a small uncertainty, $1 / \gamma^{2}$ strongly decreases and the systematic quadratic error becomes negligible.

\section{A.2. Extension of the formalism to the case of several calibrators}

An interferometric observation of a target classically needs the acquisition of a number $n$ of calibrators sequences. They can be from the same calibrator but observed before and after the target, or from several different objects of reference. For estimating the calibrated $V_{\text {target }}^{2}$, we choose to consider the weighted mean of the estimators given by each calibrator individually, the weight beeing dependent on the statistic quadratic errors only:

$$
\left\langle V_{\text {target }}^{2}\right\rangle \simeq \frac{\sum_{i=1}^{n} w_{i}\left\langle V_{\text {target }}^{2}\right\rangle_{i}}{\sum_{i=1}^{n} w_{i}}, \text { with } w_{i}=\frac{1}{\sigma_{V_{\text {target }_{\text {stat }}}^{2}}^{2}} .
$$

For the square standard deviation, taking care of always distinguish the systematic errors from the statistic ones yields a total quadratic error equal to the sum of both following terms:

$$
\begin{aligned}
\sigma_{V_{\text {target stat }}^{2}}^{2} & \simeq \frac{1}{\sum_{i=1}^{n} w_{i}}, \\
\sigma_{V_{\text {target syst }}^{2}}^{2} & \simeq \frac{\sum_{i=1}^{n} \sigma_{V_{\text {target }}^{2} \text { syst }}^{2}}{n} .
\end{aligned}
$$

Equations (A.8)-(A.10) are inserted into the VEGA postreduction tools. The latter warn the user of their limit of application: if $\beta$ or $\gamma$ is smaller than four, the user is informed that the uncertainty on respectively, the measurements or the diameter of the calibrator, is not sufficient to be confident about the value of the calibrated squared visibility. 